\title{
Fading Analysis for the High Speed Railway Viaduct and Terrain Cutting Scenarios
}

\author{
Jinghui Lu, Gang Zhu, and Bo Ai \\ State Key Laboratory of Rail Traffic Control and Safety, Beijing Jiaotong University, Beijing 100044, China \\ Correspondence should be addressed to Bo Ai, aibo@ieee.org \\ Received 27 July 2012; Accepted 12 November 2012 \\ Academic Editor: César Briso Rodríguez
}

Copyright () 2012 Jinghui Lu et al. This is an open access article distributed under the Creative Commons Attribution License, which permits unrestricted use, distribution, and reproduction in any medium, provided the original work is properly cited.

A good understanding of the fading characteristics in high speed railway environment is essential for the design of a high reliable railway wireless network. In this paper, measurements have been taken in both high speed viaduct and terrain cutting scenarios using track side base stations (BSs) of the railway wireless network in China. The measurement sites have been chosen with special care; thus the whole measured route can be characterized either by viaduct or terrain cutting. Kolmogorov-Smirnov (K-S) test has been first introduced in the statistical analysis to find out which is the most appropriate model for the small scale fading envelope. Though both Rice and Nakagami distributions provide a good fit to the first-order envelope data in both scenarios, only the Rice model generally fits the second-order statistics data accurately. For the viaduct scenario, higher Rice $K$ factor can be observed. The change tendency of the $K$ factor as a function of distance in the two scenarios is completely different. It can be concluded that the over bridges which span the railway tracks in terrain cutting scenario would affect the Rice $K$ value severely.

\section{Introduction}

As an international standard for railway communication and applications, GSM-R (GSM for Railway) has been adopted by China as a wireless communication platform to transfer security data of train control. With the rapid development of high speed railways in China, GSM-R networks have been widely deployed along the railway lines. Since GSM$\mathrm{R}$ aims to transmit security data and information for train control, failure or obstruction of the wireless network will inevitably affect the normal running of the railway system. To provide a safe and reliable network, the design of a railway wireless network is of vital importance. In the meanwhile, appropriate wireless network design and optimization rely heavily on accurate prediction of radio wave propagation.

The propagation environment of the high speed railways is a lot different from the common public wireless network. As indicated in [1], typically a railway track is full of terrain cuttings, tunnels, bridges, and so forth. Track side base stations (BSs) are often used to provide a seamless coverage. Besides, the BS antenna is always much higher than the surrounding environment to insure that there is a lineof-sight (LOS) component between the BS antenna and the train antenna. So far, channel analysis in these special railway environments has attracted more and more research interests. The measured path loss values in viaduct scenarios have been analyzed in [2-4], respectively, and the path loss exponents have been found between 2 and 4 . A more general path loss model for viaduct scenarios has been proposed in [5], considering the influences of viaduct height and BS antenna relative height. The Rice $K$ factor in the viaduct and terrain cutting scenarios along the high speed railway was first estimated by a moment-based estimator, which employed the second and the fourth moments of the signal envelope [6]. However, statistical analysis of two momentbased estimators shows that moment-based estimator using the first and second moments of the signal envelope can be a better estimator, as it has better asymptotic performance [7]. And Rice $K$ factor as a function of distance has been studied in [8], which shows the $K$ factor decreases linearly in the distance. However, the estimation interval of the Rice $K$ factors was set to $100 \mathrm{~m}$, which we believe is too large to reflect the fast variation of the $K$ factors.

Besides, though Nakagami distribution has been widely accepted to be a very good fit to mobile radio channel characteristics $[9,10]$, it has never been studied for the 


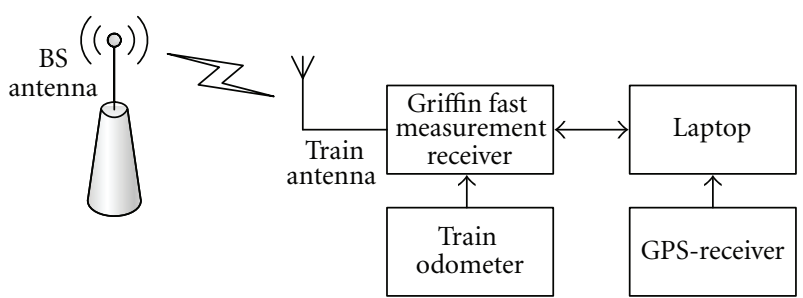

FIgURE 1: Measurement system.

small scale envelope characteristics in railway scenarios. The objective of this work is to fill this gap by investigating the suitability of Nakagami distribution for describing the small scale fading envelope. Moreover, second-order statistics of the received envelope, such as the level crossing rate (LCR) and average fade duration (AFD), have not been fully investigated. Although recent work compared the measured second-order statistics with the theoretical values of Rayleigh, Rice, and Nakagami models in viaduct scenario [11], no paper has reported the results of terrain cutting scenario. These statistics provide useful information for the statistic analysis of burst errors and the design of errorcorrecting codes.

This rest of the paper is organized as follows. A brief description of the measurement system and scenarios is given in Section 2. Theoretical models for the small scale fading channel are described in Section 3. Section 4 shows the measurement results of the two scenarios, including the envelope distribution and second-order statistics. Conclusions are drawn in Section 5.

\section{Measurement Setup}

The narrowband measurements were done along the Zhengzhou-Xi' an high-speed railway of China with a special test system, which can collect signal level data from GSM$\mathrm{R}$ track side BSs. During the measurements, the train was moving at a high speed of about $277-300 \mathrm{~km}$ per hour. The measurement system in the train periodically recorded the signal power value every 10 centimeters. Two typical viaduct and terrain cutting scenarios have been selected for analysis. And the measurements have been done twice in two different days to eliminate the effect of the measurement error. The following sections describe the measurement system and the measurement scenarios in greater detail.

2.1. Measurement System. The measurement system shown in Figure 1 consists of a receive antenna mounted on the top of the train, a Willteck 8300 Griffin fast measurement receiver, a train odometer, a global positioning system (GPS) device, and a laptop to record the test data. The measurement receiver operated in the distance trigger mode. So the train odometer was able to send a trigger signal to the receiver at intervals of 10 centimeters. A software installed in the laptop can store the measured data. And geographic location data of the measured samples can be provided by the GPS device. Table 1 shows some measurement parameters of the two
TABLE 1: Measurement parameters.

\begin{tabular}{lcc}
\hline Parameter & Viaduct & Terrain cutting \\
\hline Transmit power & $40 \mathrm{dBm}$ & $40 \mathrm{dBm}$ \\
Transmit frequency & $932.4 \mathrm{MHz}$ & $932.8 \mathrm{MHz}$ \\
Transmit antenna height & $23 \mathrm{~m}$ & $33 \mathrm{~m}$ \\
Transmit antenna gain & $17 \mathrm{~dB}$ & $17 \mathrm{~dB}$ \\
Receive antenna height & $3.5 \mathrm{~m}$ & $3.5 \mathrm{~m}$ \\
Receive antenna gain & $0 \mathrm{~dB}$ & $0 \mathrm{~dB}$ \\
Average train speed & $78.9 \mathrm{~m} / \mathrm{s}$ & $82.0 \mathrm{~m} / \mathrm{s}$ \\
\hline
\end{tabular}

scenarios. The transmit antennas are both directional and cross-polarized. The receive antennas are omnidirectional.

2.2. Measurement Scenarios. The two chosen scenarios are shown in Figure 2. The viaduct scenario depicted in Figure 2(a) seems a very good propagation environment, as there are few obstacles and scatters. A satellite image of the measured viaduct is also displayed in Figure 2(b), which shows the whole measurement route from BS ZX1706D to BS ZX1705D. During the measurements, BS ZX1706D was transmitting signal. However, the terrain cutting scenario displayed in Figure 2(c) seems more complex for radio wave propagation. Steep slopes on both sides of the railway tracks may block the radio wave or produce multiple reflections. The slopes covered with grass have a height of about 78 meters. The degree of inclination is about 70 degree. A satellite image of the measured terrain cutting is also displayed in Figure 2(d). Five over bridges which span the railway tracks can be observed in this figure. These bridges are built to facilitate traffic on the two sides of the terrain cutting. Later the effect of the over bridges on radio propagation will be illustrated. BS ZX1714D was transmitting signal during the test. The whole measurement area from BS ZX1714D to BS 1713D can be characterized by terrain cutting scenario. In both scenarios, the railway tracks are almost straight and the BS antennas are situated 17 and $37 \mathrm{~m}$ away from the railway tracks.

\section{Theoretical Models}

Various theoretical models have been presented to describe the small scale fading behavior of the mobile channel. In this section, both the envelope distribution and second-order statistics of the Rayleigh, Rice, and Nakagami fading models are discussed.

3.1. Envelope Distribution. The Rayleigh probability density function (PDF) is given by [12]

$$
p(r)=\frac{r}{\sigma^{2}} \exp \left(-\frac{r^{2}}{2 \sigma^{2}}\right) \quad(0 \leq r \leq \infty) .
$$




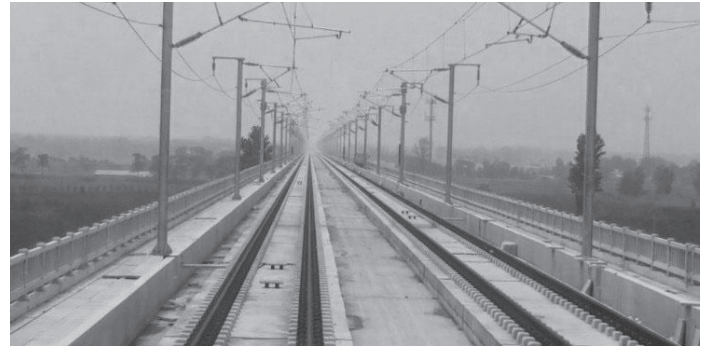

(a) Viaduct scenario

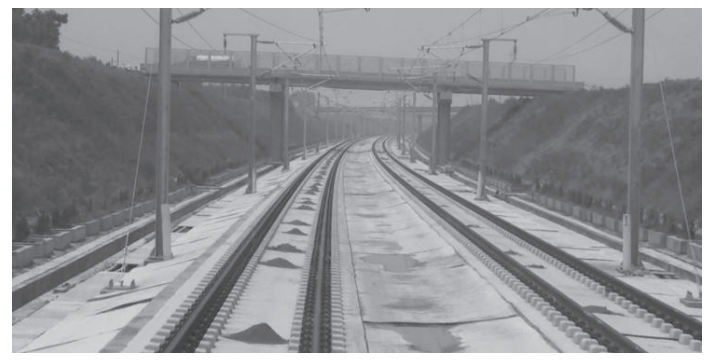

(c) Terrain cutting scenario

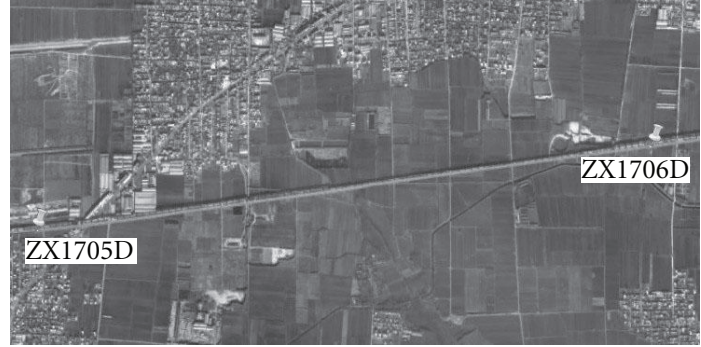

(b) Satellite image of measured viaduct

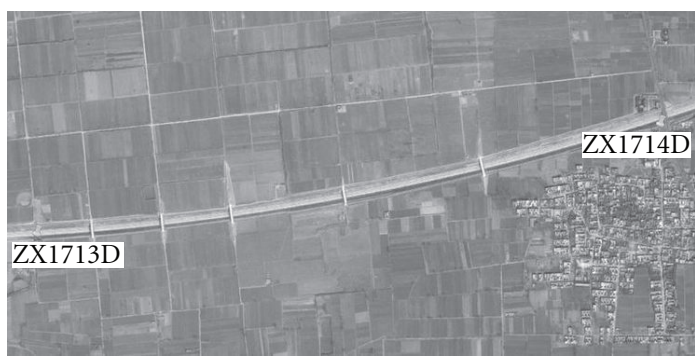

(d) Satellite image of measured terrain cutting

Figure 2: Measurement scenarios. $\sigma$ is

And the maximum likelihood estimate of the parameter

$$
\sigma_{e}=\sqrt{\frac{1}{2} E\left(r^{2}\right)}
$$

where $E\left(r^{2}\right)$ is the second moments of the measured samples.

The Rice PDF is expressed as

$$
p(r)=\frac{r}{\sigma^{2}} \exp \left(-\frac{r^{2}+s^{2}}{2 \sigma^{2}}\right) I_{0}\left(\frac{r \cdot s}{\sigma^{2}}\right) \quad(s \geq 0, r \geq 0)
$$

where function $I_{0}$ is the modified Bessel function of the first kind and zero-order. The Rice distribution is often described in terms of a parameter $K$ which is defined as the ratio between the direct or strong component power of the signal and the variance of the multipath. It is given by

$$
K=\frac{s^{2}}{2 \sigma^{2}}
$$

The parameter $K$ is known as the Rice factor and completely specifies the Rice distribution. Different methods have been proposed for estimating the Rice $K$ factor. Though the maximum likelihood estimator (MLE) can yield optimal results, it is relatively cumbersome and time consuming [13]. A simple $K$ estimation method based on the moments of the receiver envelope has also been proposed. It has been validated by [7] that moment-based estimator using the first and second moments of the signal envelope has a better asymptotic performance, compared to the one using the second and fourth moments of the envelope. The estimator based on the first and second moments of the signal envelope is used in this paper, though it is a little complex.
The moments of the Rice distribution can be expressed as [7]

$$
\begin{aligned}
\mu_{n} & =E\left[R^{n}(t)\right] \\
& =\left(\sigma^{2}\right)^{n / 2} \Gamma\left(\frac{n}{2}+1\right) \exp (-K)_{1} F_{1}\left(\frac{n}{2}+1 ; 1 ; K\right),
\end{aligned}
$$

where ${ }_{1} F_{1}(\cdot ; \cdot ; \cdot)$ is the confluent hypergeometric function, and $\Gamma(\cdot)$ is the gamma function. To derive $K$, define the following functions of $K$

$$
f_{n, m}(K)=\frac{\mu_{n}^{m}}{\mu_{m}^{n}} \quad(n \neq m)
$$

Since $f_{n, m}(K)$ depends only on $K, K$ can be derived by inverting the corresponding $f_{n, m}(K)$. When $n=1$ and $m=2$, (6) can be calculated using (5) as

$$
f_{1,2}(K)=\frac{\pi e^{-K}}{4(K+1)}\left[(K+1) I_{0}\left(\frac{K}{2}\right)+K I_{1}\left(\frac{K}{2}\right)\right]^{2} .
$$

From $(6), f_{1,2}(K)$ can also be expressed as

$$
f_{1,2}(K)=\frac{\mu_{1}^{2}}{\mu_{2}}
$$

where $\mu_{1}$ and $\mu_{2}$ can be calculated according to the moments of the measured samples, which can be given by

$$
\widehat{\mu}_{k}=\frac{1}{N} \sum_{l=0}^{N-1} R^{k}\left(l T_{s}\right)
$$

where $R\left(l T_{s}\right)$ is the measured envelope value, $T_{s}$ is the sampling period, and $N$ is the number of available samples. The corresponding estimator $\hat{K}_{1,2}$ can be obtained by combining 
(7) and (8). However, this involves the complex numerical procedure of inverting (7). A simple way to solve the problem is to implement a lookup table. To be specific, first create a lookup table by calculating all the $f_{1,2}$ values for a predefined range of $K$ values according to (7). Then compare the $f_{1,2}$ results from (8) with the values stored in the lookup table. At last the $K$ value which gives the least deviation of $f_{1,2}$ is chosen as the estimated $K$ factor.

The Nakagami PDF of the envelope $r$ is given by

$$
p(r)=\frac{2 m^{m} r^{2 m-1}}{\Gamma(m) \Omega^{m}} \exp \left(-\frac{m r^{2}}{\Omega}\right),
$$

where $m$ is the Nakagami parameter defined as the ratio between $\Omega$ squared and the variance of the envelope squared

$$
m=\frac{\Omega^{2}}{\operatorname{Var}\left(r^{2}\right)}
$$

and $\Omega$ is the average power, given by

$$
\Omega=E\left[r^{2}\right] \text {. }
$$

The Gamma function is given by

$$
\Gamma(m)=\int_{0}^{\infty} x^{m-1} \exp (-x) d x .
$$

As is generally well accepted, the parameter $\Omega$ can be reasonably estimated by

$$
\widehat{\Omega}=N^{-1} \sum_{i=1}^{N} R_{i}^{2}
$$

where $N$ is the number of available samples $R_{i}$ of the envelope. On the contrary, the shape parameter $m$ can be estimated using different methods. In [14], the performance of the inverse normalized variance, Tolparev-Polyakov, and the Lorenz estimators have been compared through Monte Carlo simulation. And the inverse normalized variance estimator has been proved to be superior to the other two estimators. Thus, it is adopted as the estimator in our paper, which is given by

$$
\hat{m}=\frac{\mu_{2}^{2}}{\mu_{4}-\mu_{2}^{2}} .
$$

3.2. Second Order Statistics. The LCR of the signal envelope reveals information about how fast the received signal changes with time. It is defined as the average number of times the signal envelope crosses a certain threshold level in a positive-going direction per second. And the AFD is defined as the average time that the fading signal envelope remains below a certain threshold level [15]. Exact formulas for second-order statistics of the Rayleigh, Rice, and Nakagami fading models have been derived in the literature and their fit to the empirical data has also been investigated in various scenarios. It is widely accepted that the goodness of fit for second-order statistics do not appear to be dependent on the accuracy of fit for first-order statistics $[16,17]$. As a result, it is essential to compare our measurement results to all of the three theoretical models, which are listed below.

For a fading signal, the LCR expressions for Rayleigh, Rice [18], and Nakagami [19] models are

$$
\begin{gathered}
N_{\text {Rayleigh }}(\rho)=\sqrt{2 \pi} f_{d} \rho \exp \left(-\rho^{2}\right) \\
N_{\text {Rice }}(\rho)=\sqrt{2 \pi(K+1)} f_{d} \rho \exp \left(-K-(K+1) \rho^{2}\right) \\
\times I_{0}(2 \sqrt{K(K+1)} \rho) \\
N_{\text {Nakagami }}(\rho)=\sqrt{2 \pi} f_{d} \frac{m^{m-(1 / 2)}}{\Gamma(m)} \rho^{2 m-1} \exp \left(-m \rho^{2}\right),
\end{gathered}
$$

where $\rho=R / \sqrt{E\left[R^{2}\right]}$ is the value of the specified level $R$, normalized to the local rms amplitude of the fading envelope, $f_{d}$ is the maximum Doppler frequency, which can be calculated by

$$
f_{d}=\frac{v}{\lambda}
$$

where $v$ is the average speed of the mobile, and $\lambda$ is the wavelength of the carrier signal. And $K$ is the Rice factor, $m$ is the Nakagami parameter. Their values can be estimated from the measured envelope samples.

The AFD formulas for Rayleigh, Rice, and Nakagami models can be expressed as

$$
\begin{gathered}
T_{\text {Rayleigh }}(\rho)=\frac{\exp \left(\rho^{2}\right)-1}{\sqrt{2 \pi} f_{d} \rho} \\
T_{\text {Rice }}(\rho)=\frac{\left[1-Q\left(\sqrt{2 K}, \sqrt{2(K+1) \rho^{2}}\right)\right]}{\sqrt{2 \pi(K+1)} f_{d} \rho} \\
\times \frac{\exp \left(K+(K+1) \rho^{2}\right)}{I_{0}(2 \sqrt{K(K+1)} \rho)} \\
T_{\text {Nakagami }}(\rho)=\frac{\Gamma\left(m, m \rho^{2}\right) \exp \left(m \rho^{2}\right)}{\sqrt{2 \pi m^{2 m-1}} f_{d} \rho^{2 m-1}} .
\end{gathered}
$$

\section{Measurement Results}

4.1. Data Processing. To determine the small scale fading statistics of the received signal envelope, the effects of the path loss and shadowing have to be removed first. In order to extract the fading envelope, the received signal is normalized to its local mean value. So, for the received sample $r\left(x_{i}\right)$, the local mean value is given by

$$
r\left(x_{i}\right)_{m}=\frac{1}{W} \cdot \sum_{i=W / 2}^{i+W / 2} r\left(x_{i}\right),
$$

where $W$ represents the number of samples in a sliding window (or bin) for the computation. This local mean value is computed for each individual sample in a bin and the normalized samples are then used for distribution fitting. As suggested by [20], a bin size of $40 \lambda$ is used for analysis, which corresponds to an average distance of approximately 
TABLE 2: K-S testing results at 5\% significance level.

\begin{tabular}{lcccc}
\hline & \multicolumn{2}{c}{ First run } & \multicolumn{2}{c}{ Second run } \\
& Viaduct & Terrain cutting & Viaduct & Terrain cutting \\
\hline Rayleigh & $19.0 \%$ & $48.8 \%$ & $20.1 \%$ & $52.5 \%$ \\
Rice & $74.7 \%$ & $85.2 \%$ & $74.7 \%$ & $93.8 \%$ \\
Nakagami & $81.0 \%$ & $89.5 \%$ & $78.9 \%$ & $92.6 \%$ \\
\hline
\end{tabular}

$13 \mathrm{~m}$. For the sampling rate used for these measurements, this distance translates to approximately 128 samples per bin.

For the first-order envelope, Kolmogorov-Smirnov (K-S) test is implemented to investigate the fitting performance of the predefined distributions for all the bins in both scenarios. It has been applied in [21-23] as a goodness-of-fit test method to verify the suitability of a hypothesized distribution and identify the distribution which best represents the experimental results.

The K-S statistic $D_{N}$, which measures the maximum deviation between the hypothesized distribution and the empirical distribution derived from the measured data, is given by [24]

$$
D_{N}=\max \left|S_{N}(x)-F(x)\right|,
$$

where $S_{N}(x)$ is the cumulative distribution function (CDF) of the empirical data, $F(x)$ is the CDF of the hypothesized distribution, and $N$ is the number of samples. The value $D_{N}$ is then compared with a critical value $J$, which is a function of significance level and the sample size $N$ [25]. If $D_{N}<J$, then the hypothesized distribution is equal to the empirical distribution. In other words, the hypothesized distribution has passed the K-S test.

4.2. Distribution Fitting Results. Table 2 shows the K-S testing results. The numerical values represent the percentages of the bins which have passed the test. It is clear that both Rice and Nakagami distribution fit the measured data very well in the two scenarios, as they both have fitted more than $74 \%$ of the bins. The differences between the fitting results of the Rice and Nakagami distribution are fairly small. On the contrary, Rayleigh distribution has only fitted a minority of tested bins. Additionally, sample plots of the empirical CDF for a particular bin and theoretical model fits are given in Figures 3 and 4 . The close resemblance between empirical distributions and the Rice and Nakagami distributions can be seen in these figures. At the same time, the Rayleigh distributions have a large deviation from the measurement data. And both the Rice and Nakagami distributions have passed the K-S tests for the bins shown here, while Rayleigh distributions have failed to pass the tests. Similar results can be observed in most of the bins. It has thus demonstrated the validity of the K-S test.

4.3. Rice K Characteristics. As the Rice model has a relatively strong physical significance, it has been selected to analyze the fading statistics in viaduct and terrain cutting scenarios. From the above test results, it can been seen that not all of the bins can be characterized as Rice distribution. Due

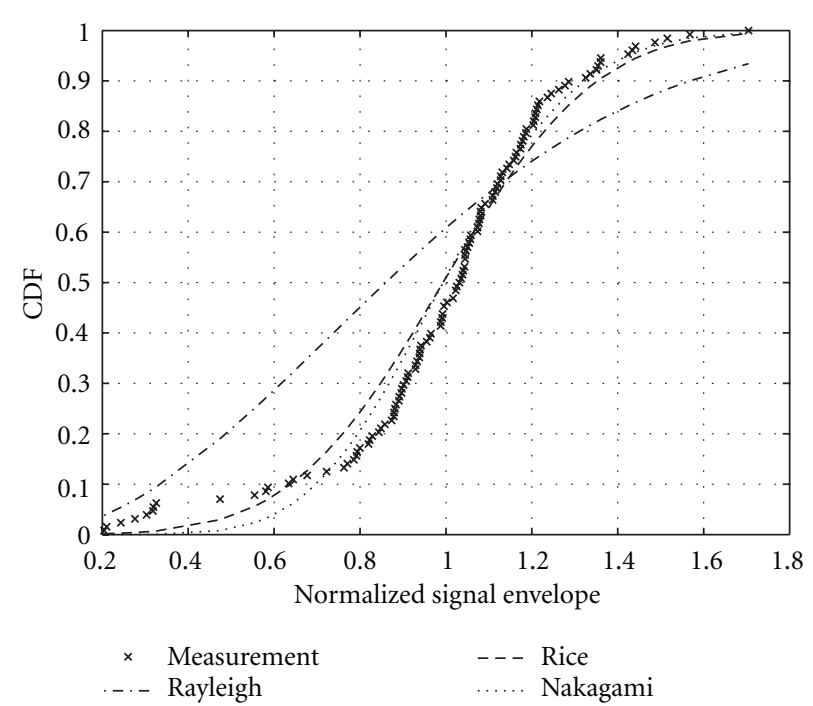

Figure 3: Sample empirical CDF of the small scale signal envelope and theoretical model fits for viaduct scenario. The Rice $K$ factor is 5.56 and Nakagami parameter $m$ is 4.37 .

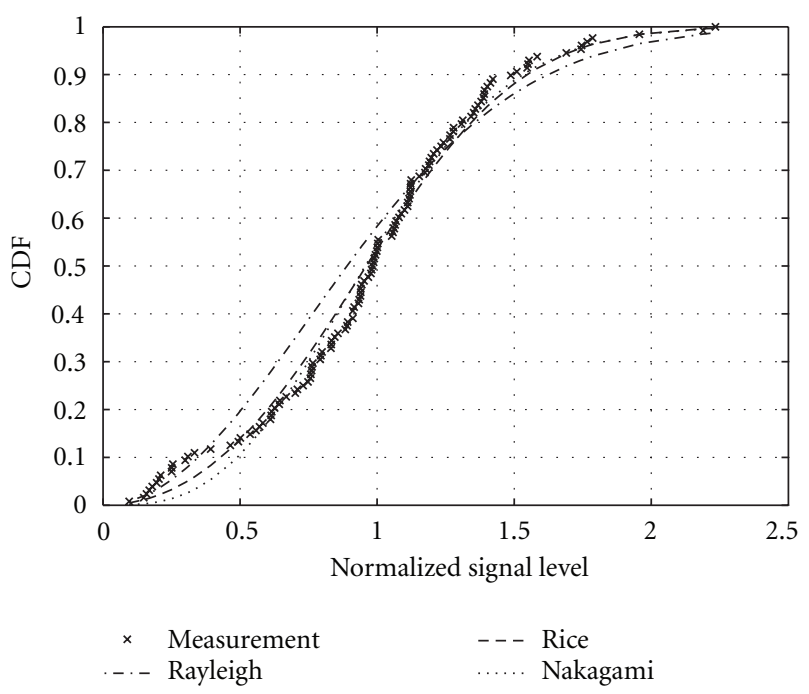

Figure 4: Sample empirical CDF of the small scale signal envelope and theoretical model fits for terrain cutting scenario. The Rice $K$ factor is 1.43 and Nakagami parameter $m$ is 1.59 .

TABle 3: Rice $K$ statistics.

\begin{tabular}{lcccc}
\hline \multirow{2}{*}{ Scenarios } & \multicolumn{4}{c}{ Rice $K$} \\
& Minimum & Maximum & Mean & Standard deviation \\
\hline Viaduct & 0 & 5.5600 & 2.3874 & 1.3314 \\
Terrain cutting & 0 & 4.9200 & 1.3390 & 1.1329 \\
\hline
\end{tabular}

to the randomness and complexity of the multipath signal components, signal envelope samples of some bins may show a complete different distribution. Thus, in order to obtain more accurate results, the estimated $K$ values which haven't passed the K-S test are discarded in the statistical analysis. Table 3 presents some statistics of the estimated Rice factors 


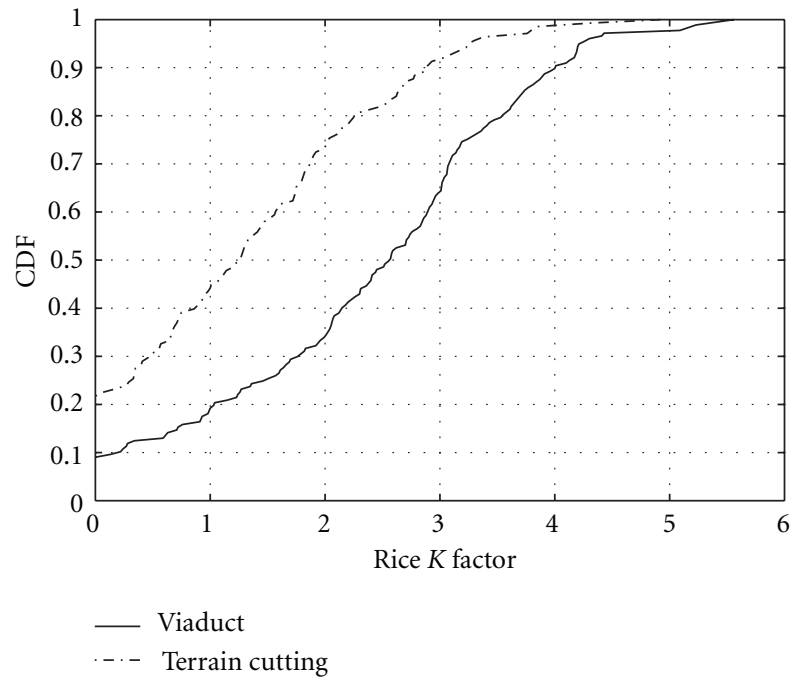

FIGURE 5: CDF of the $K$ factors in both scenarios.

(as a linear power ratio) in both scenarios, including the minimum, maximum, mean, and standard deviation values. It is seen that viaduct scenario appears to be a less severe fading channel compared to terrain cutting scenario since the mean $K$ value of the viaduct scenario is larger than that of the terrain cutting scenario. The standard deviation values of both scenarios are approximately the same.

The CDF of the obtained $K$ factors in both scenarios is also shown in Figure 5. It can be seen that for any given probability value, the $K$ value of the viaduct scenario is larger than that of the terrain cutting scenario, further confirming the relative severe propagation environment of the terrain cutting scenario. In most of the cases (about 90\%), $K$ value is within the interval 0-4 for viaduct scenario, while the value is 0-2.9 for terrain cutting scenario. In addition, approximately $22 \%$ of the $K$ values in terrain cutting scenario are zero, which implies a kind of fading as severe as Rayleigh fading. However, the proportion of zero $K$ values in viaduct scenario is only $9 \%$.

Figure 6 shows the plot of the Rice $K$ factors as a function of BS-train separation distance for both scenarios. In viaduct scenario, the Rice $K$ value tends to increase in the first few hundred meters. This could be explained by the fact that directional antennas are used in the railway wireless networks. The effect of the directional antenna is also reflected in the received signal power, which is low near the BS and gradually increases with the distance. Then the $K$ value shows a general decreasing trend. In terrain cutting scenario, the increasing trend of $K$ value for the first few hundred meters is not obvious. The reason for this is that existence of slopes may weaken the LOS component. The rest part of the Rice $K$ values does not have a regular change tendency as in the viaduct scenario. Instead, it fluctuates sharply. Locations of the five over bridges are also marked in the figure by five vertical dotted lines. It is observed that the Rice $K$ value tends to drop rapidly after passing the over bridges and then rise again. This phenomenon is extremely obvious for the last four over bridges. Thus the $K$ value

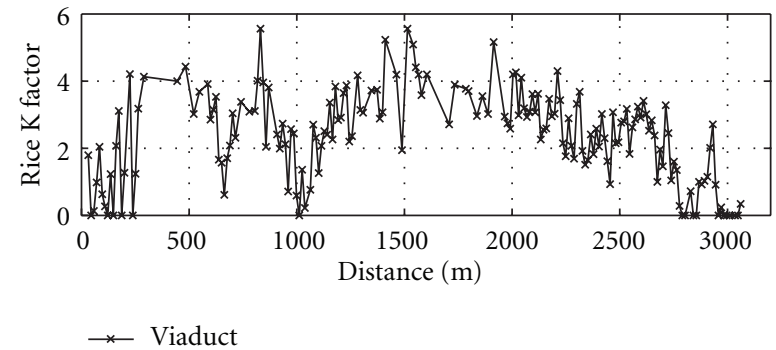

(a)

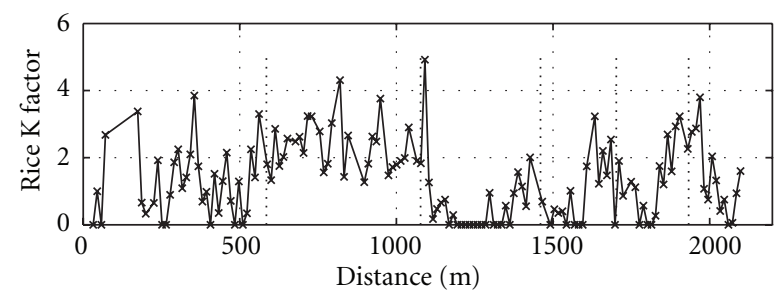

$\star *$ Terrain cutting

(b)

Figure 6: Rice $K$ factor as a function of distance.

appears to change periodically according to the existence of the bridge, while this is not observed in viaduct scenario.

4.4. Second-Order Statistics. All the obtained envelope data are first used to calculate the LCR and AFD values. After that, the measured envelope values are fitted to the theoretical distributions and then theoretical LCR and AFD values can be derived. For viaduct, the obtained Rice $K$ factor is 2.32 and Nakagami parameter $m$ is 1.99 . For terrain cutting, the Rice $K$ factor is 1.29 and Nakagami parameter $m$ is 1.50 . Both the empirical and theoretical LCR and AFD values are computed for threshold levels from $-20 \mathrm{~dB}$ to $10 \mathrm{~dB}$.

The empirical and theoretical LCR results in viaduct and terrain cutting scenario for the first measurement are shown in Figure 7. Fairly large LCR values per second can be observed in the figure. This is due to the fact that the train was moving at a very high speed. According to the theoretical formulas (16), the LCR per second increases linearly with the maximum Doppler frequency, which is a monotonic increasing function of the average speed of the mobile station. For viaduct scenario, a good agreement between the empirical LCR and Rice model can be seen for any threshold values, while a bad agreement between empirical data and the Rayleigh model can also be found. Although the Nakagami model does not give good fit for most of the threshold values, it gives excellent fit for the threshold values smaller than $-15 \mathrm{~dB}$. It is found that good match between the Nakagami and empirical CDFs does not guarantee a good match for the corresponding LCR curves. The same behavior is also obtained for the second measurement, confirming that close match for the first-order statistics may yield dissimilar second-order statistics [16]. For the terrain cutting scenario, as shown in Figure 7(b), the Rice model still gives better fit 


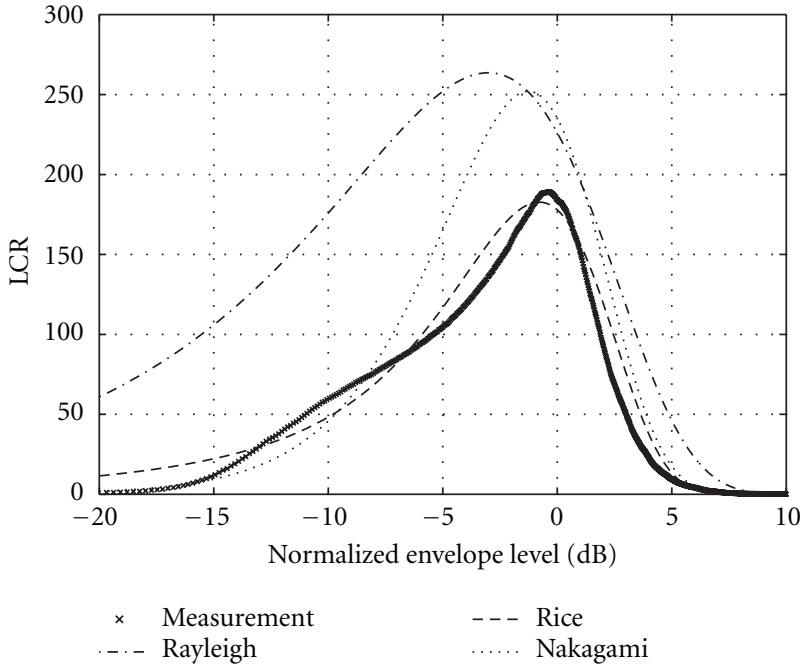

(a) Viaduct scenario

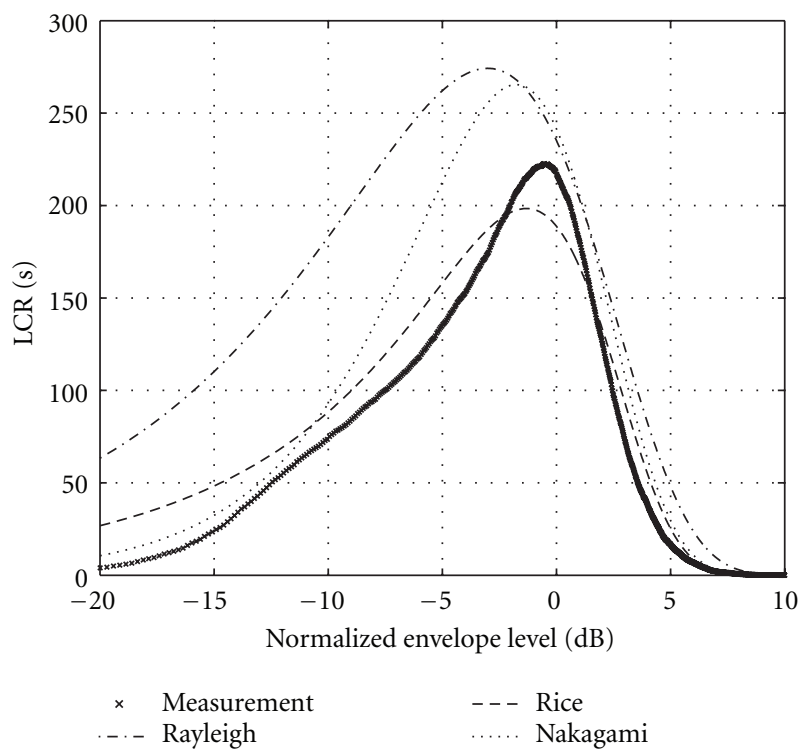

(b) Terrain cutting scenario

FIGURE 7: Empirical LCR results in both scenarios for the first measurement, together with the theoretical results of Rayleigh, Rice, and Nakagami fading models.

than the other two models. But the deviation between the empirical results and the Rice model is larger than that of the viaduct scenario, especially for the threshold value near $0 \mathrm{~dB}$. The Rayleigh model still gives the worst fit, while the Nakagami model gives a relatively better fit. Still, a relative independence of the goodness of fit between the first-order and second-order statistics can be observed.

The empirical and theoretical AFD results in viaduct and terrain cutting scenario for the first measurement are shown in Figure 8. The AFD values are plotted on a logarithmic scale to highlight the slight difference (not visible on a linear scale) between the empirical and theoretical AFDs. For both scenarios, Rice model offers the best fit for most threshold values. However, it deviates larger for the lower and upper

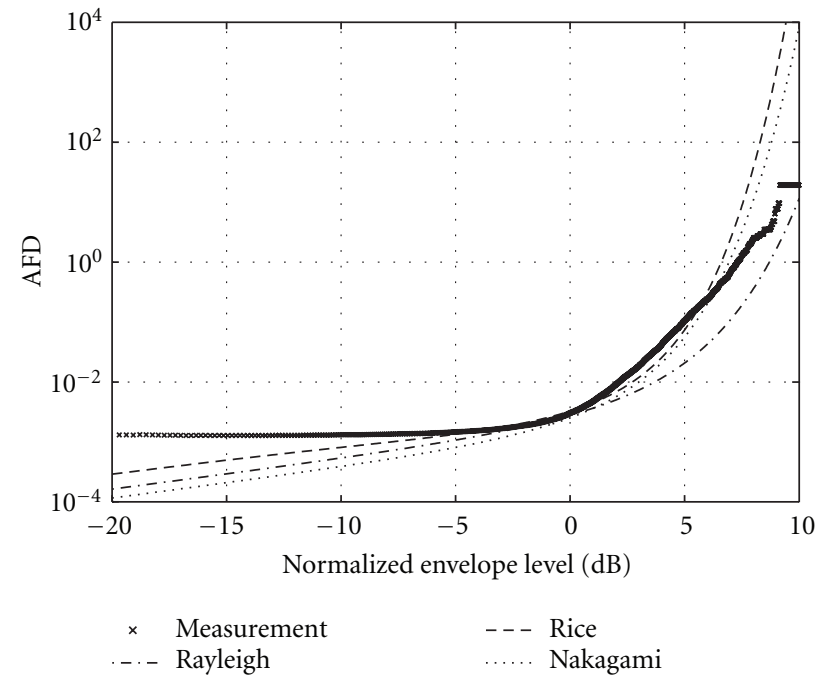

(a) Viaduct scenario

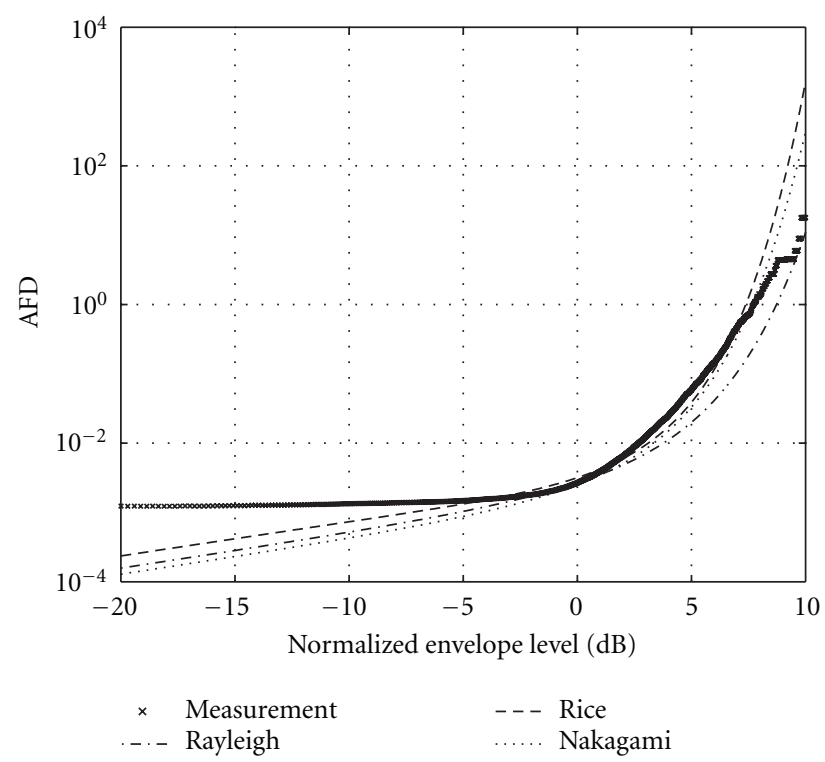

(b) Terrain cutting scenario

Figure 8: Empirical AFD results in both scenarios for the first measurement, together with the theoretical results of Rayleigh, Rice, and Nakagami fading models.

part of the curve. On the other hand, Nakagami model is more accurate for the threshold values larger than $0 \mathrm{~dB}$. The theoretical Rayleigh values are always less than the measured AFD values.

\section{Conclusion}

This paper has presented empirical fading characteristic results in typical high speed railway viaduct and terrain cutting scenarios. Measurements were done by a special test system for GSM-R. The small scale fading envelope distribution has been fitted to theoretical Rayleigh, Rice, and Nakagami distributions. K-S test has been specially introduced as a goodness-of-fit test method to verify the suitability of a 
hypothesized distribution and the results show that both Rice and Nakagami distribution can describe the empirical data very well. Statistical analysis of the Rice $K$ factors indicates that in most cases the value is within the interval 0-4 for viaduct scenario, while the value is $0-2.9$ for terrain cutting scenario. And about $22 \%$ of the Rice $K$ values in terrain cutting scenario are zero, which reveals that radio wave experiences more severe fading in terrain cutting scenario than in viaduct scenario. Further study of the Rice $K$ factor as a function of distance suggests that in viaduct scenario the $K$ value tends to increase in the first few hundred meters due to the directional antenna. Then it shows a general decreasing trend for the rest part of the distance. On the contrary, the $K$ value exhibits an irregular change tendency with distance in terrain cutting scenario. It is found that the $K$ value appears to drop and rise periodically by the effect of the over bridges which span the railway tracks. Though both Rice and Nakagami distributions provide a good fit to the first-order envelope data in both scenarios, only the Rice model generally fits the second-order statistics data accurately. This result verifies that the goodness of fit between the first-order envelope statistics and second-order statistics is relatively independent. These results will certainly enable better comprehension of the propagation channel in typical high speed railway scenarios and thus have important implications for the system design of the network.

\section{Acknowledgments}

This work is supported in part by the Joint Funds of the Fundamental Research Funds for the Central Universities under Grant no. 2010JBZ008 and 2012YJS017, State Key Program of NSFC (Grant no. 60830001), the State Key Laboratory of Rail Traffic Control and Safety (Contract no. RCS2008ZZ006), (Contract no. RCS2008ZZ007), (Contract no. RCS2010 K008), Beijing Jiaotong University. At last but not least, the authors would like to thank the anonymous reviewers for their valuable comments and suggestions to improve the quality of this paper.

\section{References}

[1] C. Briso, C. Cortés, F. J. Arqués, and J. I. Alonso, "Requirements of GSM technology for the control of high speed trains," in Proceedings of the 13th IEEE International Symposium on Personal, Indoor and Mobile Radio Communications (PIMRC '02), vol. 2, pp. 792-793, September 2002.

[2] H. Wei, Z. Zhong, K. Guan, and B. Ai, "Path loss models in viaduct and plain scenarios of the high-speed railway," in Proceedings of the 5th International ICST Conference on Communications and Networking in China (ChinaCom '10), pp. 15, August 2010.

[3] R. He, Z. Zhong, B. Ai, L. Xiong, and H. Wei, "A novel path loss model for high-speed railway viaduct scenarios," in Proceedings of the 7th International Conference on Wireless Communications, Networking and Mobile Computing (WiCOM '11), pp. 1-4, September 2011.

[4] J. Lu, G. Zhu, and B. Ai, "Radio propagation measurements and modeling in railway viaduct area," in Proceedings of the 6th International Conference on Wireless Communications,
Networking and Mobile Computing (WiCOM '10), pp. 1-5, September 2010.

[5] R. He, Z. Zhong, B. Ai, and J. Ding, "An empirical path loss model and fading analysis for high-speed railway viaduct scenarios," IEEE Antennas and Wireless Propagation Letters, vol. 10, Article ID 5981374, pp. 808-812, 2011.

[6] L. Gao, Z. Zhong, B. Ai, and L. Xiong, "Estimation of the Ricean $\mathrm{K}$ factor in the high speed railway scenarios," in Proceedings of the 5th International ICST Conference on Communications and Networking in China (CHINACOM '10), pp. 1-5, August 2010.

[7] C. Tepedelenlioğlu, A. Abdi, and G. B. Giannakis, "The Ricean K factor: estimation and performance analysis," IEEE Transactions on Wireless Communications, vol. 2, no. 4, pp. 799-810, 2003.

[8] R. He, Z. Zhong, B. Ai, and J. Ding, "Propagation measurements and analysis for high-speed railway cutting scenario," Electronics Letters, vol. 47, no. 21, pp. 1167-1168, 2011.

[9] M. Nakagami, "The m-distribution-a general formula of intensity distributionof rapid fading," in Statistical Method of Radio Propagation, 1960.

[10] H. Suzuki, "A statistical model for urban radio propogation," IEEE Transactions on Communications, vol. 25, no. 7, pp. 673680, 1977.

[11] L. Gao, Z. Zhong, B. Ai, L. Xiong, and H. Zhang, "Analysis and emulation of the small-scale fading characteristics in the highspeed rail scenarios," in Proceedings of the 6th International ICST Conference on Communications and Networking in China (CHINACOM '11), pp. 1181-1185, August 2011.

[12] T. S. Rappaport, Wireless Communications: Principles and Practice, Prentice Hall, New York, NY, USA, 2nd edition, 2002, http://www.worldcat.org/isbn/0130422320.

[13] L. J. Greenstein, D. G. Michelson, and V. Erceg, "Momentmethod estimation of the Ricean K-factor," IEEE Communications Letters, vol. 3, no. 6, pp. 175-176, 1999.

[14] A. Abdi and M. Kaveh, "Performance comparison of three different estimators for the nakagami m parameter using Monte Carlo simulation," IEEE Communications Letters, vol. 4, no. 4, pp. 119-121, 2000.

[15] B. Talha and M. Patzold, "Level-crossing rate and average durationof fades of the envelope of mobile-to-mobile fading channelsin cooperative networks under line-of-sight conditions," in Proceedings of the IEEE GlobalTelecommunications Conference (GLOBECOM '08), pp. 1-6, December 2008.

[16] A. Abdi, K. Wills, H. A. Barger, M. S. Alouini, and M. Kaveh, "Comparison of the level crossing rate and average fade duration of Rayleigh, Rice, and Nakagami fading models with mobile channel data," in Proceedings of the 52nd Vehicular Technology Conference (VTC '00), pp. 1850-1857, September 2000.

[17] M. D. Yacoub, M. V. Barbin, M. S. de Castro, and J. E. B. Vargas, "Level crossing rate of Nakagami-m fading signal: field trials and validation," Electronics Letters, vol. 36, no. 4, pp. 355357, 2000.

[18] G. Stüber, Principles of Mobile Communication, Kluwer Academic, New York, NY, USA, 1996.

[19] M. D. Yacoub, M. D. Yacoub, J. E. V. Bautista, and L. Guerra de Rezende Guedes, "On higher order statistics of the Nakagami$\mathrm{m}$ distribution," IEEE Transactions on Vehicular Technology, vol. 48, no. 3, pp. 790-794, 1999.

[20] W. C. Y. Lee, "Estimate of local average power of a mobile radio signal," IEEE Transactions on Vehicular Technology, vol. 34, no. 1, pp. 22-27, 1985. 
[21] V. Nikolopoulos, M. Fiacco, S. Stavrou, and S. R. Saunders, "Narrowband fading analysis of indoor distributed antenna systems," IEEE Antennas and Wireless Propagation Letters, vol. 2, pp. 89-92, 2003.

[22] D. Kim, M. A. Ingram, and W. W. Smith, "Measurements of small-scale fading and path loss for long range RF tags," IEEE Transactions on Antennas and Propagation, vol. 51, no. 8, pp. 1740-1749, 2003.

[23] M. Boutin, A. Benzakour, C. L. Despins, and S. Affes, "Radio wave characterization and modeling in underground mine tunnels," IEEE Transactions on Antennas and Propagation, vol. 56, no. 2, pp. 540-549, 2008.

[24] P. K. Tang, Y. H. Chew, L. C. Ong, M. K. Haldar, and L. Bin, "Small-scale transmission statistics of UWB signals for body area communications," in Proceedings of the IEEE 64th Vehicular Technology Conference (VTC '06), pp. 1-5, September 2006.

[25] B. C. Liu, K. H. Lin, and J. Y. Chen, "Ricean K-factor estimation in cellular communications using kolmogorov-smirnov statistic," in Proceedings of the Asia-Pacific Conference on Communications (APCC '06), pp. 1-5, September 2006. 

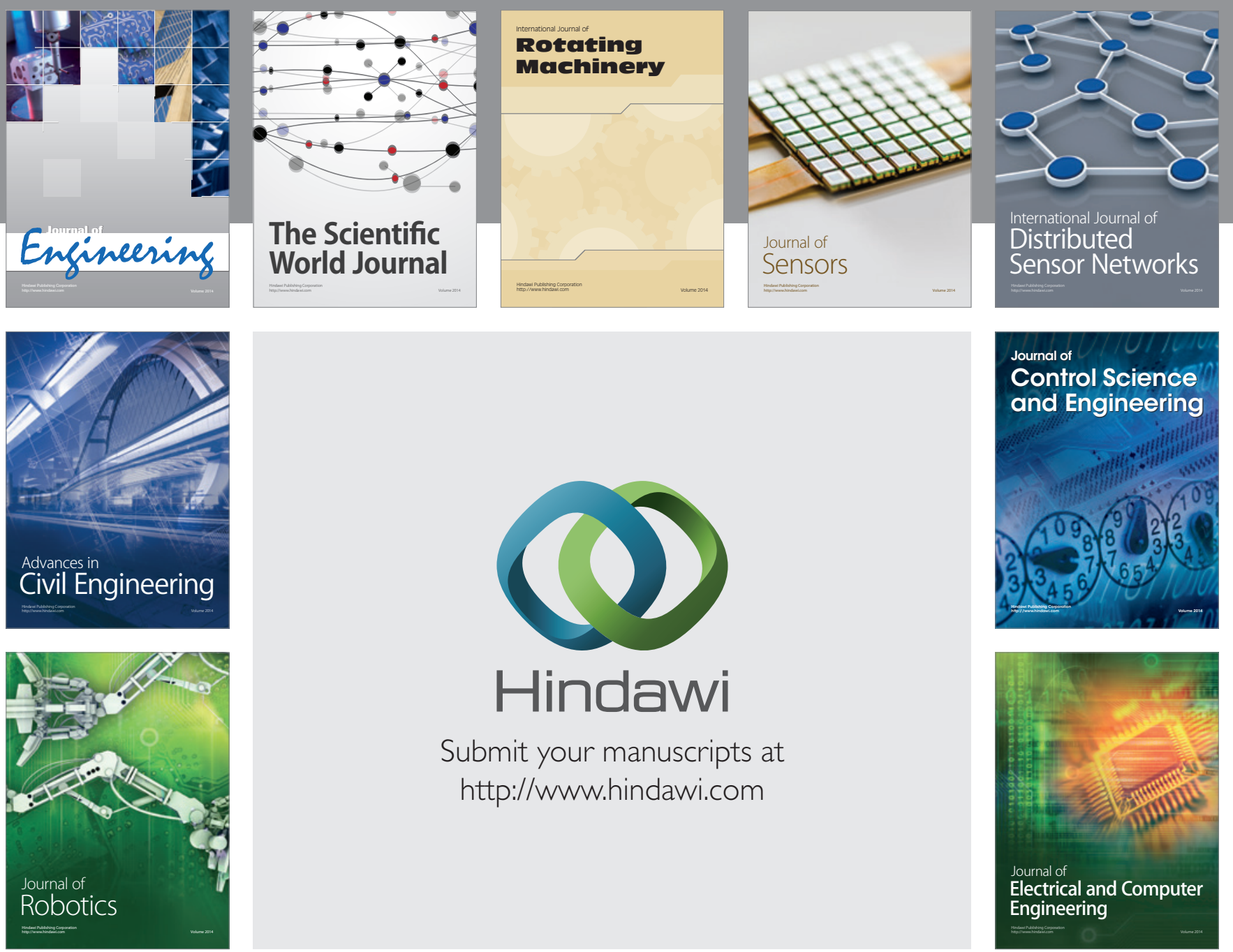

Submit your manuscripts at

http://www.hindawi.com
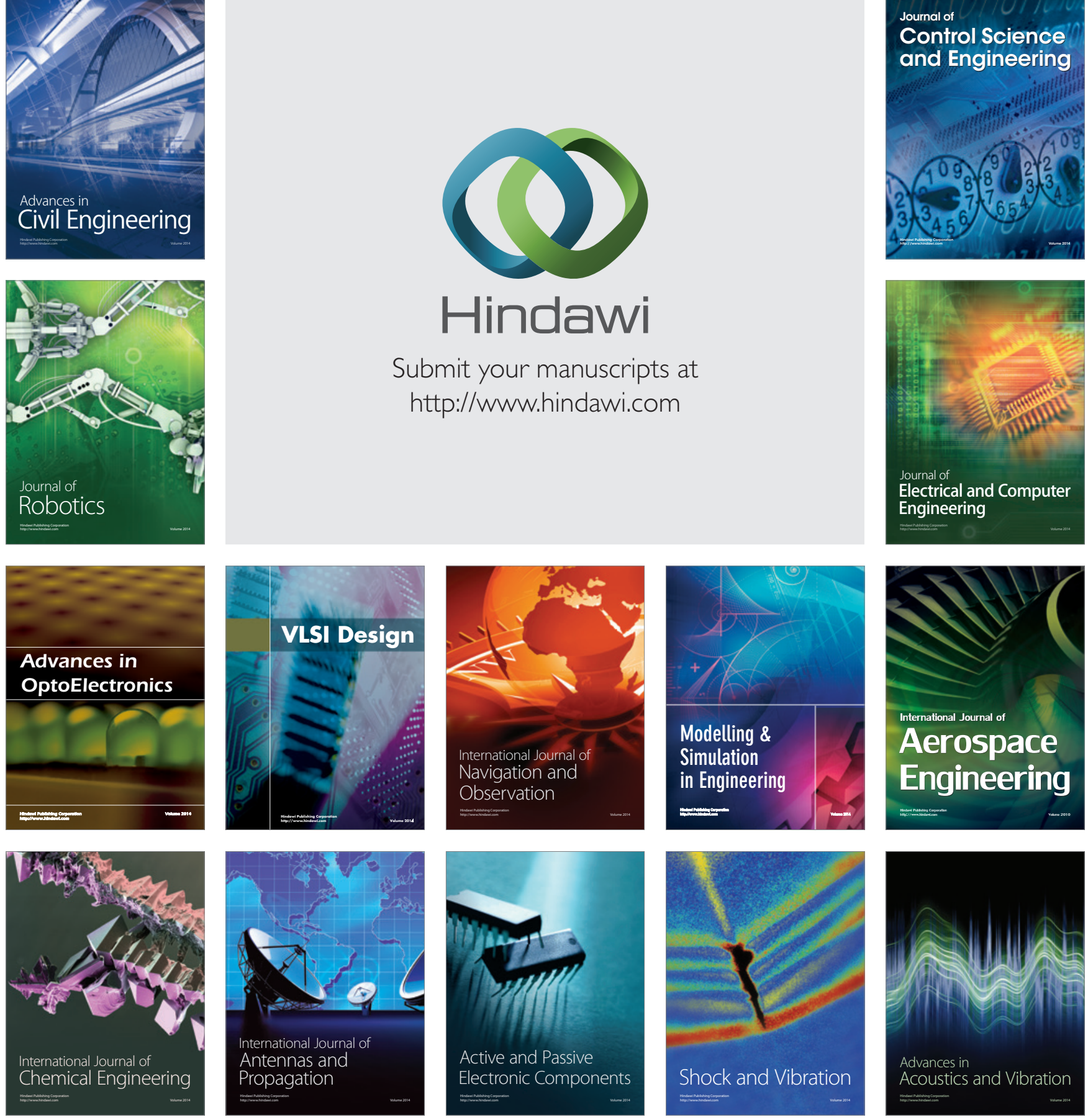